\title{
GENETIC ALGORITHMS TO DETERMINE THE OPTIMAL PARAMETERS OF AN ENSEMBLE LOCAL MEAN DECOMPOSITION
}

\author{
Willian T. F. D. Silva*, Filipe D. D. M. Borges \\ Western Paraná State University, Engineering and Exact Sciences Center, Av. Tancredo Neves, 6731, \\ 85867-970, Foz do Iguaçu/PR, Brazil \\ * corresponding author: willian.silva7@unioeste.br
}

\begin{abstract}
An optimization method for an ensemble local mean decomposition ( $E L M D)$ parameters selection using genetic algorithms is proposed. The execution of this technique depends heavily on the correct choice of the parameters of its model as pointed out in previous works. The effectiveness of the proposed method was evaluated using synthetic signals, discussed by several authors. The resulting algorithm obtained similar results to $O E L M D$, but with an $82 \%$ reduction in processing time. Actual vibration signals were also analysed, presenting satisfactory results.
\end{abstract}

KEYWORDS: Ensemble local mean decomposition, genetic algorithms, signal processing, optimization.

\section{INTRODUCTION}

Due to the wide variety of application, several signal processing techniques have been developed in recent years [1]. In 1998, a major advance in so-called decomposition techniques occurred when [1] introduced the empirical mode decomposition $(E M D)$, which was an effective tool for a non-linear and non-stationary signal analysis. In this method, a complex signal could be decomposed on a series of sums of finite functions called intrinsic mode functions $(I M F)$ that represents the oscillatory components of the signal. However, one of the main disadvantages of this technique is its susceptibility to mode mixing phenomenon. 4]. Mode mixing occurs when multiple modes resides withing one $I M F$. To prevent this, in 2005, the local mean decomposition $(L M D)$ [2] was developed in order to mitigate the mode mixing.

Nevertheless, when tested against highly complex and contaminated signals, such as faulty mechanical components vibration, despite showing superiority when compared to EMD [5], the $L M D$ still suffers, in a prohibitive way, of mode mixing problem. Therefore, in order to improve its applicability in complex signals [3] proposed an ensemble local mean decomposition $(E L M D)$, which adds white noise to the vibration signals in order to obtain the optimal compositions. Meanwhile, according to 4, the effectiveness of ELMD in reducing mode mixing is highly influenced by its parameters, such as white noise amplitude, bandwidth and ensemble numbers. Different drawbacks are also pointed out by other authors, such as the occurrence of pseudo-components ([6]) and poor signal reconstruction $([7])$.

Regarding the choice of appropriate parameters for the $E L M D, 4$ proposed an optimized ensemble local mean decomposition (OELMD), an optimization of the technique, in which parameters are chosen to satisfy the decomposition performance. However, the technique used a gross-based method for testing of several values for the parameters, leading to a highly prohibitive computational cost.

In this scope of optimization, genetic algorithms $(G A)$ are techniques that search for the best result based on the principles of genetics and natural selection strongly studied by [8-15] and disseminated since 1970s. A $G A$ allows that a population composed of various individuals get involved in certain rules that minimize (or maximize) a cost function. This article proposes a new approach in the optimization of ELMD parameters to satisfactorily fulfill the decomposition performance. Thus, better results than the ELMD with regards to mode mixing are expected, as well as better results than $O E L M D$ in terms of processing time.

Although this article is inspired by previous works [4], 16], none of these studies, unlike ours, reported the use of genetic algorithms to reduce the number of iterations necessary in the search for optimal parameters..

Furthermore, in order to assess the effectiveness of the proposed method in one of its many applications, this work intends to use the algorithm in the analysis of bearing failure diagnosis.

Thus, this work comes with the following contributions: (a) development of a new procedure based on genetic algorithms to determine white noise parameters in an ELMD; (b) extend the work of 4 on the investigation of RRMSE and $S N R$ in the optimal parameters selection; and (c) evaluation of the effectiveness of time-frequency techniques in the diagnosis of fault mechanical components.

The work is divided into six sections: Section 1 introduces the subject of decompositions and forms of optimization; Section 2 - introduces the fundamentals of local mean decomposition and its derivations; 
Section 3- presents the methods of optimization of parameters based on genetic algorithms; Section 4 compares the proposed methodology with that suggested by [4] and other LMD improvements, by means of a synthetic signal; Section 5- proposes a methodology for improving the decomposition results by reapplying the technique based on the value of RRMSE; Section 6- shows the results of a test performed on real vibrational data; and Section 7- conclusion.

\section{LOCAL MEAN DECOMPOSITION}

Assuming that a signal can be represented as the sum of a finite set of a product function from which it is possible to extract instantaneous frequency imbued with physical meaning, 2 developed a method that basically consists in decomposing the signal in several other functions, obtained from the product between an envelope signal and a frequency modulated signal, from which it is possible to extract the instantaneous frequency, thus it can be represented as a time function and as a frequency function, giving a rise to timefrequency representation (TFR).

In the method, the signal decomposition is performed by a progressive decoupling of the frequency modulated signal from an amplitude modulated envelope, throughout the following steps:

(1.) Obtaining all the local extremes from the signal $x(t)$. The extreme indices are denoted by $e^{k}$ and the correspondent extremes by $x\left(e^{k}\right)$

(2.) Calculating the smoothed local mean $m(t)$ and smoothed local amplitude $a(t)$. To acquire these values two preliminary steps are necessary. The first one, characterized by the calculation of the preprocessed local mean $m^{0}(n)$ and the local amplitude $a^{0}(n)$, it is obtained by:

$$
\begin{aligned}
& m^{0}(n)=\frac{x\left(e^{k}\right)+x\left(e^{k+1}\right)}{2} \text { for } e^{k} \leq n \leq e^{k+1} \\
& a^{0}(n)=\frac{\left|x\left(e^{k}\right)-x\left(e^{k+1}\right)\right|}{2} \text { for } e^{k} \leq n \leq e^{k+1}
\end{aligned}
$$

However, despite the simplicity and consistency of equations, [17] warn that results can not be obtained without the extension of the signal, which may introduce disagreements in its ends that gradually influence its middle, disturbing the decomposition performance [18]. Thus, the authors proposed a treatment for the extremes, called a boundaries processing method that calculates the local mean and local amplitude, by means of the following equations.

For the first extreme:

$$
\begin{gathered}
m^{0}(n)=\frac{x\left(e_{1}\right)+2 x\left(e_{2}\right)+x\left(e_{3}\right)}{4} \\
a^{0}(n)=\frac{\left|x\left(e_{1}\right)-x\left(e_{2}\right)\right|+\left|x\left(e_{2}\right)-x\left(e_{3}\right)\right|}{4}
\end{gathered}
$$

For the last one:

$$
\begin{aligned}
& m^{0}(n)=\frac{x\left(e_{M-2}\right)+2 x\left(e_{M-1}\right)+x\left(e_{M}\right)}{4} \\
& a^{0}(n)= \\
& =\frac{\left|x\left(e_{M}\right)-x\left(e_{M-1}\right)\right|+\left|x\left(e_{M-1}\right)-x\left(e_{M-2}\right)\right|}{4}
\end{aligned}
$$

Where $M$ is the signal length. Moreover, [19] determined a signal extension algorithm that modifies the extremes by a spline interpolation, which is based on the work of [20].

Afterwards, from the variables $m^{0}(n)$ and $a^{0}(n)$, the smoothed local mean $m(t)$ and smoothed local amplitude $a(t)$ are obtained. There are differences in how this smoothing is calculated. Although the method proposed by [2], using the moving average algorithm $(M A)$, has been studied with proven efficacy [3, 5, 18, 19, 21, [22] argue that this method could lead the decomposition to incoherent results. Thus, 23] proposed a cubic spline interpolation based on $L M D(S L M D)$ due its property of a good convergence and high smoothing. However, [24] confirmed that large interpolation errors can occur in the local amplitude calculation. Thereby, the authors have proposed a rational Hermite interpolation $(O L M D)$, replacing the spline interpolation, stating that it could better counteract the waveform of the amplitude. Nevertheless, 22 ] confirm that the Hermite interpolation cannot adaptively adjust the shape of the curves with the varying local characteristics of the waveform in the sifting process. Therefore, the authors suggest that a rational spline Interpolation coupled with an optimization procedure of a tension parameter could control the shape of the cubic spline. According to the authors' studies, their method yields more accurate results of decomposition as well as a reduction of the total processing time of the technique. Some smooth examples are show in Figure 1 .

(3.) Calculate the estimated zero-mean signal $h_{11}$ and FM signal $s_{11}$ by means of the variables $x(n), m(n)$ and $a(n)$. For that, the equation is defined by:

$$
\begin{gathered}
h_{11}(n)=x(n)-m_{11}(n) \\
s_{11}(n)=\frac{h_{11}(n)}{a_{11}(n)}
\end{gathered}
$$

It is important to make sure that $s_{11}(n)$ is a purely FM signal. Otherwise, the function $x(n)$ assumes the value of $s_{11}(n)$ and the steps are repeated until the condition described by Equation 9 is satisfied. This condition is the so-called sifting process.

$$
\lim _{p \rightarrow \infty} a_{1 p}(n)=1
$$




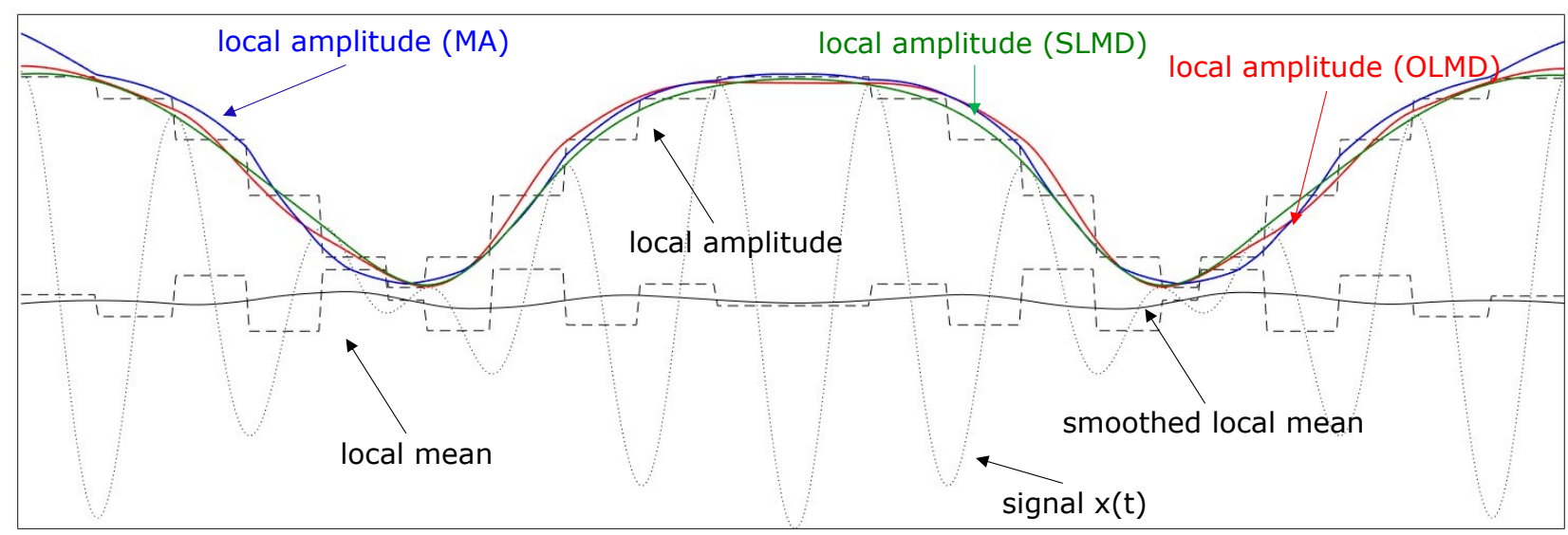

FiguRE 1. Hypothetical signal $x(t)$ together with the local mean, smoothed local mean, local amplitude and local amplitude smoothed by $M A, S L M D$ and $O L M D$ methods.

Due to its notorious importance in the $L M D$ final results [22, [19] proposed a method called sifting stopping, which defines an optimal number of iterations for the decomposition, which consequently brought better results of the method as well as reducing the processing time.

(4.) Calculation of the signal $s_{1}(n)$, envelope signal $a_{1}(n)$ and the product function $P F_{1}(n)$ after the execution of the sifting process. Considering a process with $\mathrm{p}$ iterations, the values of $s_{1}(n), a_{1}(n)$ and $P F_{1}(n)$ are given by:

$$
\begin{gathered}
s_{1}(n)=s_{1 p}(n) \\
a_{1}(n)=\prod_{j=1}^{N} a_{i j}(n) \\
F_{1}(n)=a_{1}(n) \cdot s_{1}(n)
\end{gathered}
$$

(5.) Subtract the product function from the signal $x(n)$. The process must be repeated $\mathrm{m}$ times until the entire signal is decomposed, so that:

$$
x(n)=\sum_{i=1}^{m} P F_{i}(n)
$$

\subsection{ENSEMBLE LOCAL MEAN DECOMPOSITION}

Components of the product function with different characteristics are obtained by means of the $L M D$ method. However, due to the signal discontinuity, mode mixing still occurs during the $L M D$ process. This condition causes an ambiguity in the physical meaning of the instantaneous frequencies of the product function after the decomposition.

From this point of view, [3] demonstrated that the addition of different Gaussian white noises to the signal prior to its decomposition by $L M D$ could drastically decrease the mode mixing phenomenon. Although it may seem that the addition of the disturbance to the signal could reduce the signal-to-noise ratio $(S N R)$ and consequently bring erroneous results to the decomposition, due to the addition of a nonexistent product function, the authors proved that because there are several independent Gaussian white noises, the average of all added noise would tends to zero. Thus, the technique repeatedly applies the $L M D$ method to the signal along with a Gaussian white noise of a finite amplitude. The average of the product functions derived from the various applications is used as the result of the decomposition. Since the mean noise is zero, all disturbance added can be considered as excluded. This technique was called ensemble local mean decomposition (ELMD) and yields results far superior to those of $L M D$ in a fault diagnosis of rotating machines [3, 21, 25].

According to [26] ELMD can be described by the following steps:

(1.) Adding white noise to the signal $x(t)$ thus forming $y(t)$.

(2.) Applying the $L M D$ to signal $y(t)$ in order to obtain multiple product functions.

(3.) Repeat steps 1 and 2 several times adding different noises at each iteration.

(4.) Calculating the mean of the PF obtained and consequently using it as the result of the decomposition.

\section{ENSEMBLE LOCAL MEAN DECOMPOSITION BASED ON GENETIC ALGORITHMS}

Genetic algorithm $(G A)$ is based on the principles of genetics and natural selection. A $G A$ allows a 
population composed of many individuals to be involved under some sort of natural selection rule, so that the final population is the one that best fits those conditions.

Thus, the first step is to define the condition of the environment for the population, in this case, the cost function. In this work, it is essential that that the white noise added to the original signal has a maximum value of $R R M S E$, which evaluates the difference between the product functions and the original signal in order to cancel the mode mixing. However, the cost function minimization is used as an optimization notation, thus establishing the cost function as:

$$
\text { Cost }=-R R M S E
$$

Therefore, an initial population is defined, which can be formed by totally random chromosomes or by initial guesses in order to improve the convergence of the algorithm. In the proposed method, the population is defined in a totally random way, but within a defined range based on data taken from the signal to be decomposed.

$$
\text { Population }=\operatorname{rand}\left(N_{\text {cro }}, N_{\text {var }}\right)
$$

Where, $N_{\text {cro }}$ represents the number of chromosomes of a population and $N_{v a r}$ represents the number of alleles contained in the chromosome.

Subsequently the pairing is defined, where the most adapted chromosomes are placed in order to cross. Parents are defined randomly, and each pair produces two descendants, which contain traits from each parent. The parents still survive to be a part of the next generation. The more similar the two parents are, the better is the convergence towards a final population.

Once the pairing is defined, the crossover stage is started. Several methods have been developed to optimize the creation of a descendant. The simplest methods of crossbreeding are those called [11] points. In these, one or more chromosome points are selected as crossing points. Then, the variables between these points are exchanged between the two parents. The main disadvantage of this technique is that there is no new information in the generation of individuals, they are just replicas of the random values provided by the initial population. Therefore, a variation of this method was suggested, the so-called simple crossing [12]. In this method, a descendant comes from the combination of parents, so the chromosome assumes new values, even though it is still related to its predecessors. The formation of an allele for this chromosome is demonstrated by:

$$
\begin{array}{r}
\text { allele }_{\text {new }}=\alpha \cdot \text { allele }_{\text {mom, }, n}+ \\
+(1-\alpha) \cdot \text { allele }_{d a d, n}
\end{array}
$$

$\alpha$ being a random number between 0 and 1 ; allele $_{m o m, n}: n^{\text {th }}$ allele on the mother chromosome, and allele dad,n $: n^{\text {th }}$ allele on the father chromosome. Note that in a simple crossing, if the value of $\alpha=0.5$, the descending chromosome allele becomes a simple average of the parent variables. However, even if this method allows new information to be entered by combining information from parents, it does not allow values outside parent's extremes. Therefore, another approach, proposed by [12], was the heuristic crossover, which again uses a random variable, $\beta$, chosen in the interval [0.1], to define one or more alleles of the descendants. In this work, the use of the heuristic crossover was defined in the implementation of the algorithm because it yields better results in the search for the global maximum [13. Thus, in the generation of a new population lineage, this crossing is imposed for at least one allele of each descendant. This one is chosen at random and the remaining variable is fairly distributed to the children, so that each parent is always represented.

$$
\begin{array}{r}
\text { allele }_{\text {new }}=\beta \cdot\left(\text { allele }_{\text {mom }, n}-\right. \\
\text { allele } \left._{\text {dad }, n}\right)+ \\
+ \text { allele }_{m o m, n}
\end{array}
$$

Finally, some form of mutation can be defined for chromosomes in the population. The mutation process is important in some cases where a function can assume several local maximums and the cost function eventually converges to one of these maximums. If there is no preventive measure, the result can be far from the overall maximum cost. In this work, the mutation is defined in a completely random way, where a random value between 0 and 1 is calculated. If it is greater than 0.8 (arbitrarily chosen value) a chromosome is recalculated in a random way, without presenting any correlations with its parents.

The proposed method is exemplified by the flowchart shown in Figure 2, which represents an application of genetic algorithms to the method developed by 3$]$.

\section{Simulated signal Test}

The test of the proposed technique was carried out using a synthetic signal $x(t)$ extracted from [4, obtained by summation of the three components presented in Equations 18, 19 and 20 shown in Figure 3. Since it is often impossible to know all the compositions of a real signal, the use of synthetic ones is very useful for the evaluation of a signal processing method.

$$
\begin{gathered}
x(t)=x_{1}(t)+x_{2}(t)+x_{3}(t) \\
x_{1}(t)=1.5 \cdot e^{-800 t^{\prime}} \cdot \sin (2 \pi \cdot 5000 t) \\
x_{2}(t)=0.2 \cdot(1+\cos (2 \pi \cdot 100 t)+\cos (2 \pi \cdot 100 t)
\end{gathered}
$$




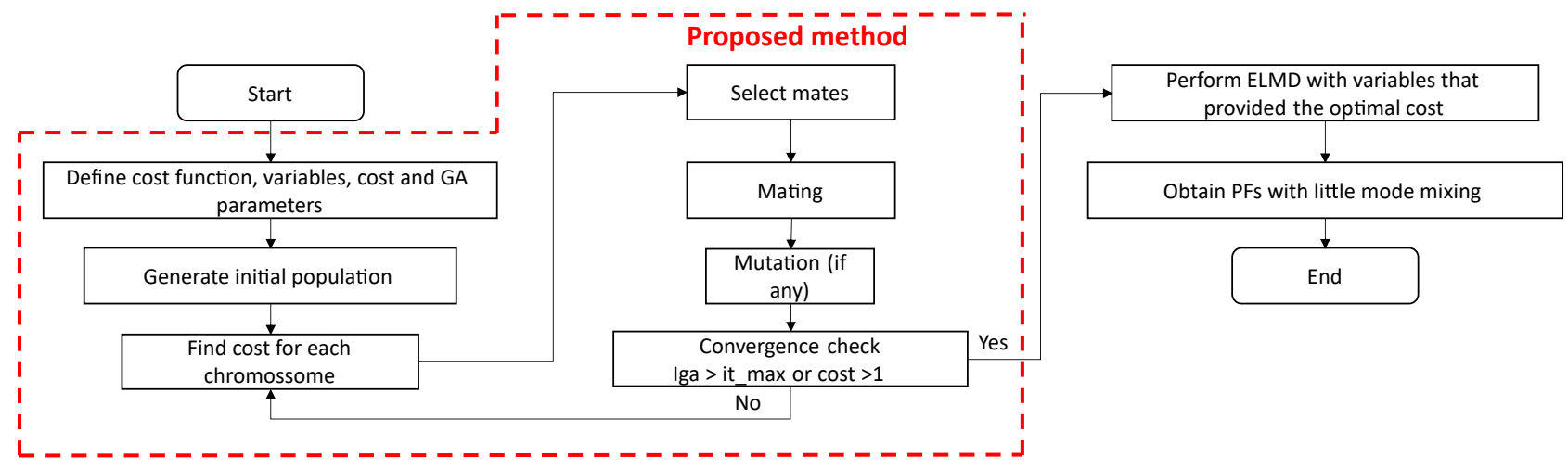

FIGURE 2. Flowchart of the proposed method based on genetic algorithms for parameter selection.

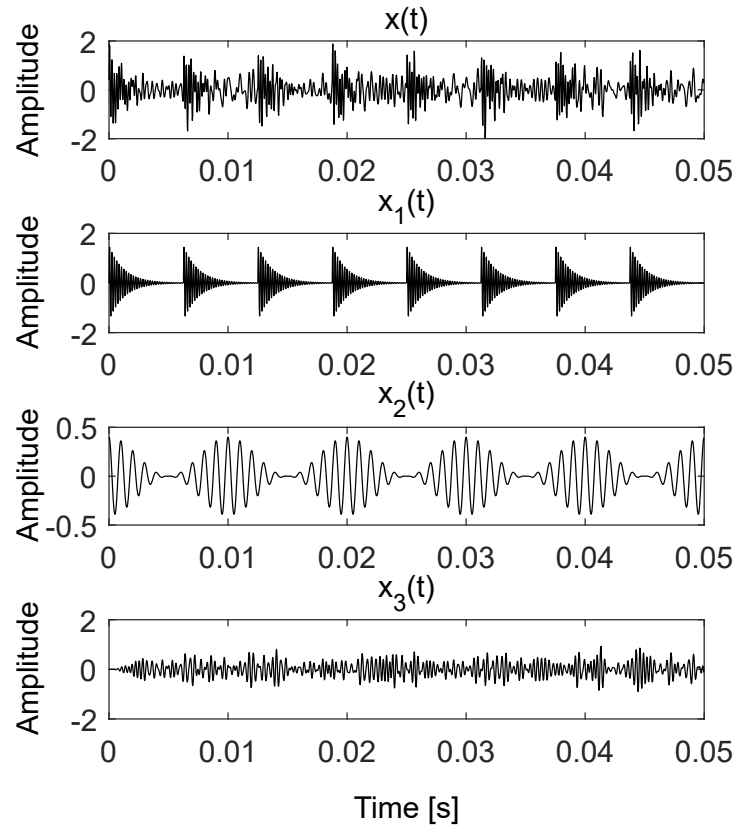

Figure 3. Waveform in the time domain of the signal $x(t), x_{1}(t), x_{2}(t)$ and $x_{3}(t)$.

Where, $x_{3}(t)$ is white Gaussian noise with a bandwidth from 2 to $4 k H z$, and; $t^{\prime}$ is a periodic function with a fundamental period of $1 / 160 \mathrm{~s}$. According to [4, this frequency was chosen because, when compared with low frequency noises, high frequencies generally present larger contributions to the extremes of the original signal.

In order to compare the performance of the proposed GAB-ELMD (genetic algorithm based - ensemble local mean decomposition) to OELMD and $L M D$ methods (using $M A$ and sifting process for smoothing, respectively) root-mean-square error $(R M S E)$, the number of product functions and processing time are considered as indicators. The expression for the $R M S E$ is given by.

$$
R M S E=\sqrt{\frac{\sum_{i=1}^{N}\left[x_{i}(t)-P F_{i}(t)\right]^{2}}{N}}
$$

Where $x_{i}(t)$ and $P F_{i}(t)$ are the original components of the signal and its decomposed form, respectively. A lower $R M S E$ value indicates a better performance. The computer used for the simulation is a $2.4 \mathrm{GHz}$ i7-Dual Core processor with 8 GB RAM. The software used is MATLAB (R2018b). The tests were performed ten times and the results shown in Table 1 represent their means.

Values presented in Table 1 shows similar results for both methods, making it impossible to point out the best within the giving confidence interval. In terms of the computational cost, OELMD required a longer time due to its test characteristic, while the proposed technique obtained similar results with a shorter average processing time without significant losses in quality of the signal, highlighting a reduction in processing time of $82.6 \%$.

The processing time is also illustrated in Figure 5 . which displays the relationship between the number of samples of a discrete signal and the processing time of each of the techniques shown in Table 1 Results are shown along their error calculated by a Student's tdistribution for a confidence interval of $95 \%$, with the proposed technique being superior to the $O E L M D$ in each instance. As for the decomposition error, it can be seen that although the proposed method had better average values as compared to $O E L M D$, it shows higher error values due to its inherent characteristic of finding adjustable results not always aligned with the global maximum, which corroborates the data presented in Figure 6, which displays a heat map containing the number of times that a given solution arranged in the model of Figure 4 was achieved by the technique based on the genetic algorithm, in a test performed one hundred times. Figure shows that there were many cases where an overall maximum was not reached.

\section{Proposed ALgorithm FOR IMPROVEMENT OF DECOMPOSITION RESULTS}

After the signal is decomposed by GAB-ELMD, some product functions still contain mode mixing, which can be observed in the previous section. Although it is 


\begin{tabular}{ccccc}
\hline \multirow{2}{*}{ Methods } & \multicolumn{3}{c}{ RMSE } & Processing \\
\cline { 2 - 4 } & PF1 & PF2 & PF3 & Time (s) \\
\hline OELMD & $0,171 \pm 0,017$ & $0,125 \pm 0,006$ & $0,198 \pm 0,013$ & 166,5 \\
GAB & $0,197 \pm 0,033$ & $0,117 \pm 0,020$ & $0,190 \pm 0,028$ & 28,9 \\
\hline
\end{tabular}

TABLE 1. Comparison of performance between OELMD's and proposed method's performances.

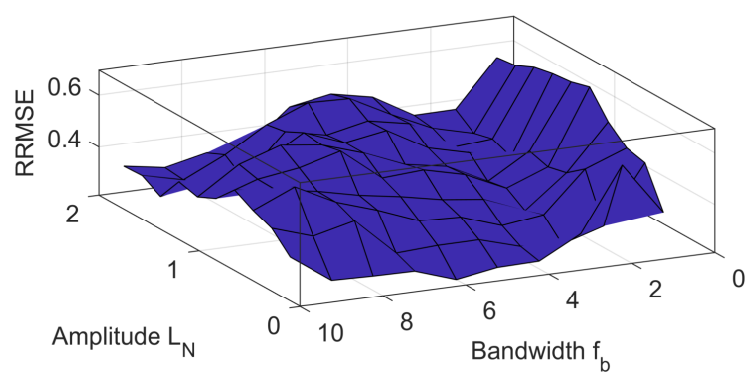

FiguRE 4. Model of the RRMSE values for signal defined by Eq. 18 based on the amplitude and bandwidth values of the noise.

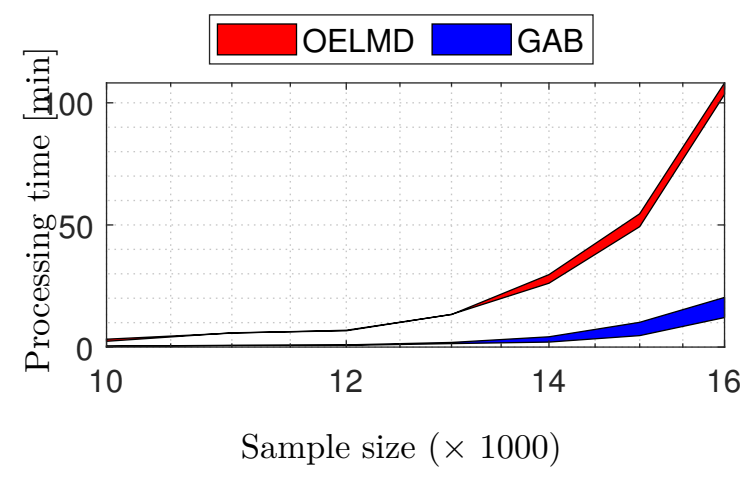

FiguRE 5. Signal's processing time per sample size.

an intrinsic phenomenon of the ELMD method, in this work, the reapplication of the decomposition based on $R R M S E$ in order to mitigate mode mixing is proposed. Recent works, such as [16] and [4, have already investigated the use of $R R M S E$ as a parameter of mode mixing between product functions, but none of them used it in order to reapply the decomposition in the product functions with the highest RRMSE. Based on the characteristics of this function, reapplication of decomposition is based on the following steps:

(1.) $G A B-E L M D$ is initially applied to decompose the signal.

(2.) The RRMSE matrix is then calculated making it possible to compare the relative root-mean-square error between the product functions. In this way, it is expected to find the product function with a higher mode mixing (minimum value).

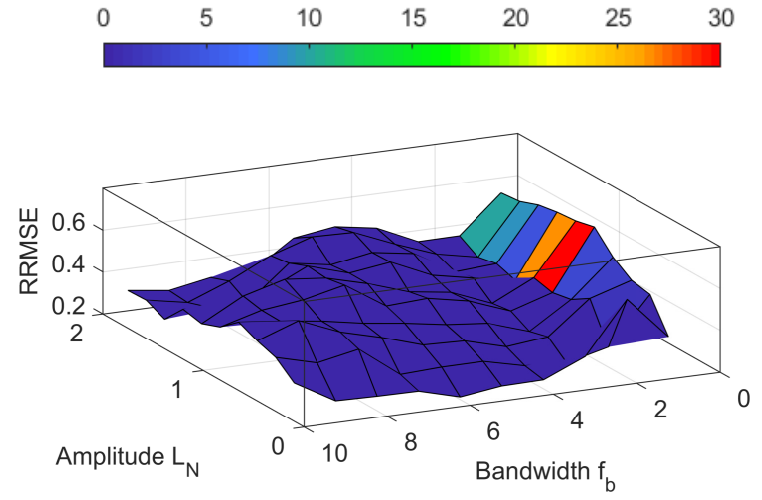

Figure 6. Heat map showing the number of times such a solution was obtained for a method based on genetic algorithm.

$$
\mathbf{M}=\left(\begin{array}{ccc}
R M S E\left(P F_{i}, P F_{i}\right) & \cdots & R M S E\left(P F_{i}, P F_{j}\right) \\
\vdots & \ddots & \vdots \\
R M S E\left(P F_{j}, P F_{i}\right) & \cdots & R M S E\left(P F_{j}, P F_{j}\right)
\end{array}\right)
$$

(3.) The PF with the highest mode mixing is selected.

(4.) $L M D$ is applied to the PF previously selected.

(5.) Each new product function is compared through the RRMSE with the PFs obtained in step 1. The $P F$ of step 1 with the closest resemblance to the new one will be added to it or be replaced by it (if the $P F$ is selected in the third step).

(6.) The procedure is repeated from step 2 to 5 until the lowest value in the $R R M S E$ array is greater than or equal to 1 , or the number of desired maximum iterations is reached.

(7.) Gets the product functions with the least mix of modes.

In order to compare the effectiveness of the proposed method, it was applied to a hypothetical signal defined by Equations 18$] 20$, which was previously decomposed by the methods mentioned in Section 2 Thus, the PFs obtained (Figures 7,11) were again compared to the respective components of the original signal obtained by means of $R M S E$. The values are then presented in Table 2 built by performing each algorithm 10 times. Data shows the RMSE average calculated along with its error, using a Student's t-distribution for a $95 \%$ confidence level. 


\begin{tabular}{ccccc}
\hline \multirow{2}{*}{ Method } & \multicolumn{3}{c}{ RMSE } & $\begin{array}{c}\text { Number } \\
\text { of } P F s\end{array}$ \\
\cline { 2 - 4 } & PF1 & PF2 & PF3 & \\
\hline SLMD & $0.331 \pm 0,000$ & $0.134 \pm 0,000$ & $0.301 \pm 0,000$ & 3 \\
\hline OLMD & $0.331 \pm 0,000$ & $0.134 \pm 0,000$ & $0.302 \pm 0,000$ & 3 \\
\hline ILMD & $0.211 \pm 0,000$ & $0.086 \pm 0,000$ & $0.302 \pm 0,000$ & 4 \\
\hline OELMD & $0,171 \pm 0,017$ & $0,125 \pm 0,006$ & $0,198 \pm 0,013$ & 3 \\
\hline Proposed & $0.154 \pm 0,062$ & $0.101 \pm 0,072$ & $0.172 \pm 0,0485$ & 3 \\
\hline
\end{tabular}

TABLE 2. Comparison of performance between proposed algorithm and methods discussed in Section 2
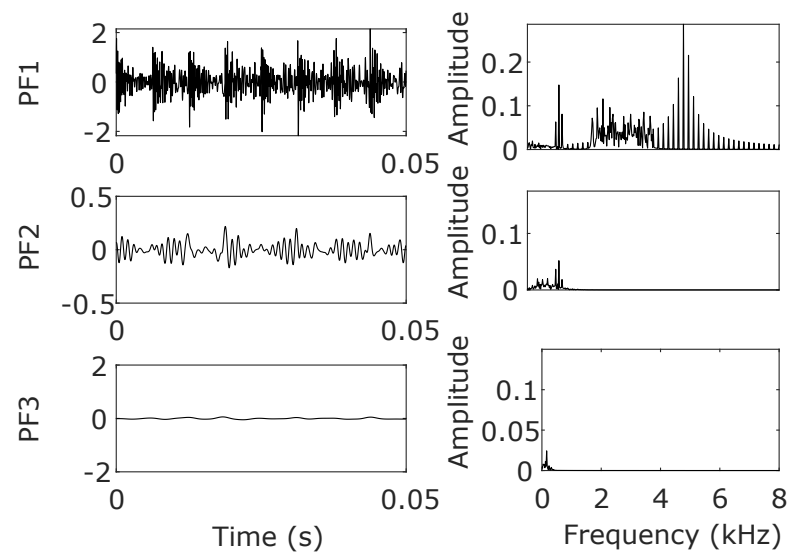

Figure 7. Signal in time domain (left) and frequency domain (right) by $S L M D$ method.
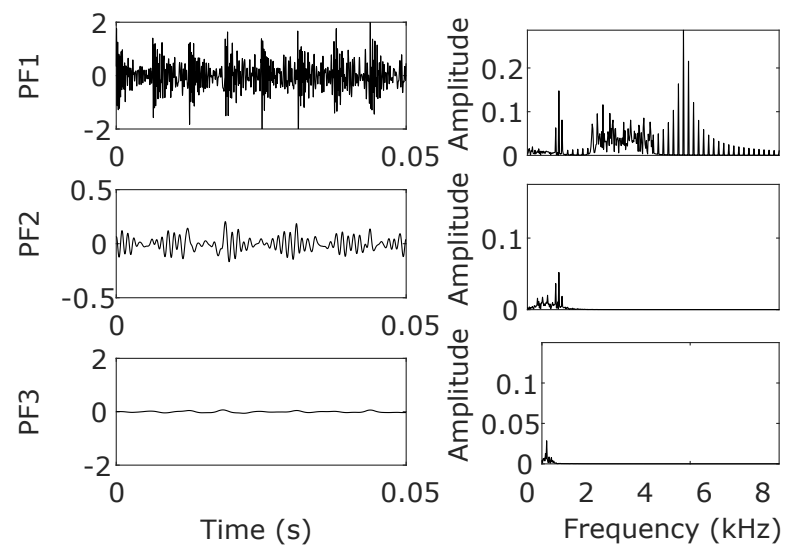

Figure 8. Signal in time domain (left) and frequency domain (right) by $O L M D$ method.

The figures show that all methods discussed in Section 2 presented a major problem of mixing modes. Figures 7 and 8 shows that the SLMD and OLMD methods had almost the same result being unable to separate the signal into its product functions, with almost the entire signal restricted in the first product function. Although the second PF still keeps its signal characteristics, the amplitudes are very low. In the same manner, PF3 shows its noise characteristics, depicting it more like an error function. For $I L M D$ (Fig. 9), the best results for $P F 2$ are evident, clearly
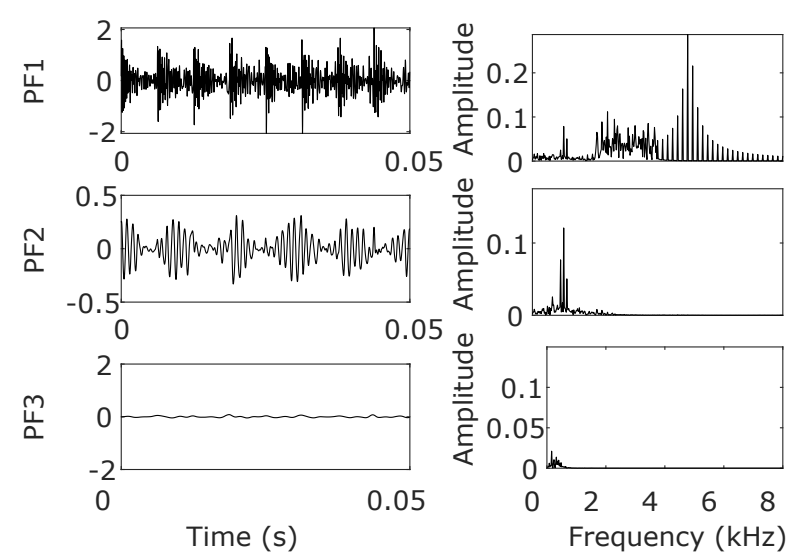

Figure 9. Signal in time domain (left) and frequency domain (right) by ILMD method.
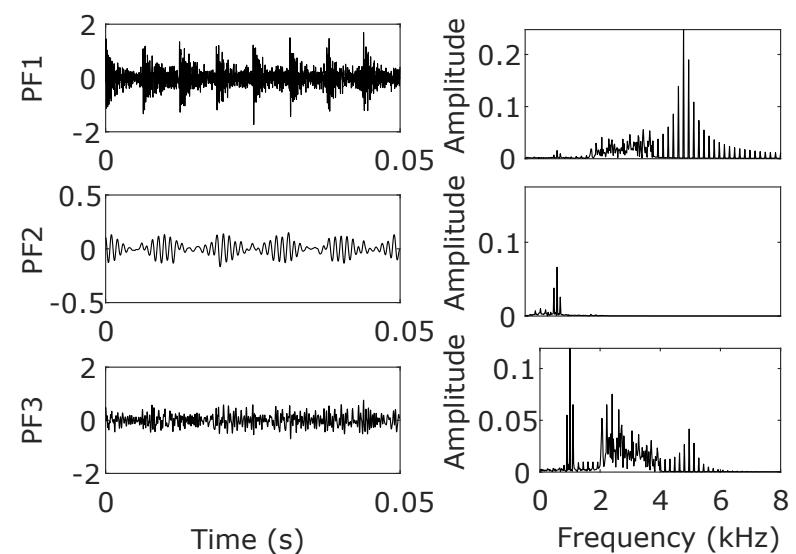

Figure 10. Signal in time domain (left) and frequency domain (right) by OELMD method.

showing the beating signal with amplitude levels very close to the original, however, the method was unable to separate the impact signal from the noise, basically leaving the two compositions only in PF1. As for OELMD (Fig. 10), even though the optimal result shown by $I L M D$ for $P F 2$ was not reproduced, it was quite efficiently able to separate the noise, leaving the product function representing the impact signal alone at PF1. However, the mode mixing still remained, as much of the beating signal was embedded in the noise signal. Finally, the proposed method managed 

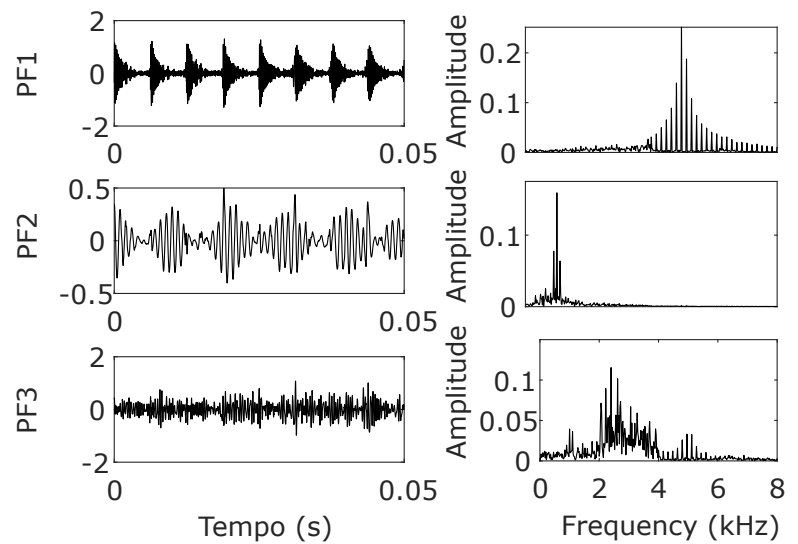

Figure 11. Signal in time domain (left) and frequency domain (right) by proposed method.

to separate the signal into three product functions very similar to the original composition, with the noise being isolated in only one function, the beating signal with optimum amplitude levels in another and the first product function representing only the impact signal.

Table 2 shows the same results as Figures 7 -11. however, numerically highlighting that the proposed method gives better average results than all the algorithms tested for PFs 1 and 3. For PF2, the $I L M D$ method showed better results, as in some cases the proposed method was not able to improve the beating signal decomposition, having similar results to the ones presented by $O E L M D$. Another fact to mention are the high variances presented by $O E L M D$ and the proposed method against the null variance of the other algorithms. This is because, as already mentioned, the optimal bandwidth and noise amplitude values to be applied to the decomposition are not always the same, which causes fluctuations in the decomposition results.

\section{EXPERIMENTAL DATA ANALYSIS}

To assess the proposed algorithm in a real case scenario, a set of rolling bearing data obtained from a test rig was used and the results were compared. Figure 12 shows the experimental test rig and all the apparatus used in the test while their specifications and technical and instrumental characteristics are shown in Table 3. Two model 6004-2RS1 (SKF) bilaterally shielded rolling bearings were used, their dimensions and construction data are shown in Table 4

From the data presented in Table 4, the characteristic fault frequencies on the outer and inner races have been calculated and presented in Table5. The damage to the bearings was made by means of a $1 \mathrm{~mm}$ diameter diamond-tipped drill mounted on a mini electric bench driller. The localized damage has approximate dimensions of $1.2 \mathrm{~mm}$ in diameter and $1 \mathrm{~mm}$ in depth. After the defect was imposed, the steel chips from

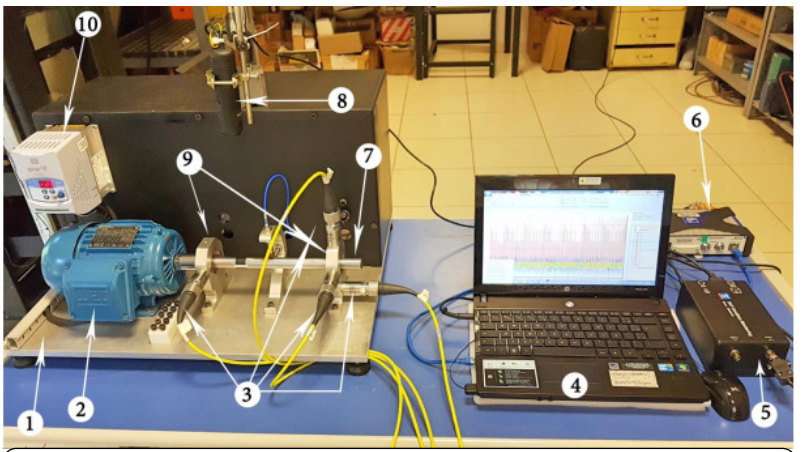

1) base, 2) drive motor, 3) accelerometers, 4) computer, 5) data conditioning, 6) amplifier, 7) drive shaft, 8) optical encoder, 9) bearing, 10) frequency inverter

Figure 12. Test rig.

\begin{tabular}{ll}
\hline Equipment & Specifications \\
\hline \multirow{3}{*}{ Drive motor } & Weg W22 - TFVE \\
& Nominal speed: $2985 \mathrm{rpm}$ \\
& Power: 1.0 kW \\
\hline \multirow{2}{*}{ Frequency inversor } & Weg CFW10 \\
& Power: $1.5 \mathrm{~kW}$ \\
\hline \multirow{3}{*}{ Amplifier } & HBM Quantum X \\
& Sample frequency: $19200 \mathrm{~Hz}$ \\
\hline & SKF $739 \mathrm{~L}$ \\
& Sensibility: $500 \mathrm{mV} / \mathrm{g} \pm 5 \%$ \\
& Frequency range: \\
& $- \pm 5 \%: 0.6-700 \mathrm{~Hz}$ \\
Accelerometer & $- \pm 10 \%: 0.4-1000 \mathrm{~Hz}$ \\
& $- \pm 3 \mathrm{~dB}: 0.2-2300 \mathrm{~Hz}$ \\
\hline
\end{tabular}

TABLE 3. Specifications and technical characteristics of the test rig.

the damaged components were removed, the proper lubricant reapplied and shielding plates reassembled.

Therefore, the vibration signal was decomposed using the proposed and the OELMD methods, obtaining a series of PFs and a constant residual. Then, an envelope analysis was applied to the second PF to identify the localized defect on the bearing.

\subsection{BeARING With OUTER RACE FAULT}

Figure 13 shows the envelope spectrum of the signal for the outer race defect, being (a) without any preprocessing treatment; (b) using the OELMD for the pre-processing; (c) using the proposed method for the pre-processing. As seen, in (a), although the characteristic bearing failure frequencies are seen, they are not evidenced and are hidden in noises of an order of magnitude similar to the excitations. In (b), although presenting different spectrum, the noise is still dominant and has even worsened the visualization of the characteristic failure frequencies. Thus, only in the method proposed in (c), it is possible to clearly visualize the $B P F O$ frequency and its harmonics, clearly showing a failure signal. 


\begin{tabular}{ll}
\hline Construction feature & Dimension \\
\hline Outer diameter & $42 \mathrm{~mm}$ \\
Inner diameter & $20 \mathrm{~mm}$ \\
Rolling's element diameter & $6.35 \mathrm{~mm}$ \\
Number of rolling elements & 9 \\
\hline
\end{tabular}

TABLE 4. Geometry parameters of the experimental bearing.

\begin{tabular}{ll}
\hline Fault frequency & Value $(\mathrm{Hz})$ \\
\hline BPFI & 269,7 \\
BPFO & 178,0 \\
\hline
\end{tabular}

TABle 5. Bearing's fault frequencies.

\subsection{Bearing With inner RaCe FAUlt}

In a similar way to outer race signals, the envelope spectra for the inner race are shown in Figure 14

Unlike the previous case, it was already possible to observe the characteristic fault frequencies without the presence of a signal pre-processing. Although the decomposition techniques removed a significant amount of noise level from the analysis, the proposed algorithm, for instance, could not clearly show the third harmonic.

\section{Conclusions}

Ensemble local mean decomposition is a new method in time-frequency analysis which comprises the main innovation of the $L M D$, being the separation of a single component $A M-F M$ signal into a set of product functions of the envelope signal, and a purely frequency-modulated signal, with a significant improvement in mode mixing. However, an intensive search for parameters that actually solve the problem is required, which can be time and human resource consuming. In this work, an approach using a wellknown optimization method was suggested in order to select the parameters for ELMD automatically and with the least time consumption. At this stage, the results demonstrate the superiority of the proposed technique to the OELMD, leading to the following conclusions:

(1.) Although OELMD shows a greater selectivity of the parameters, the proposed technique presented similar results when applied on a synthetic signal.

(2.) The proposed method showed lower processing time, reducing the total time by more than $82 \%$.

(3.) It was found that the main difficulty in achieving better processing times in the execution of the technique was the smoothing algorithm, discussed in Section 2 delineating a new field of research.

(4.) Even though the results are promising, they have not been compared to other new techniques that address $E L M D$ limitations in other ways, focusing

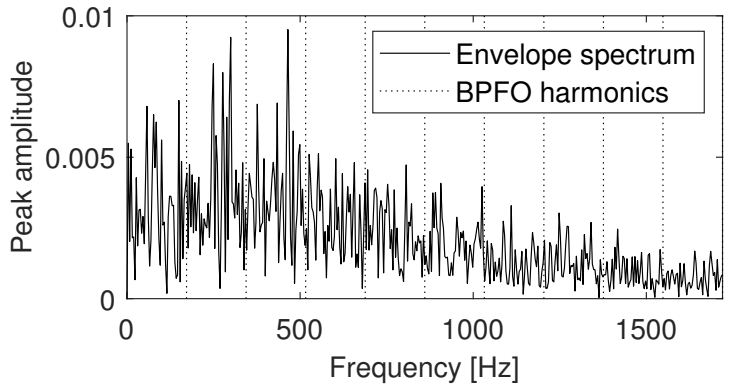

(A) . Without any pre-processing method.

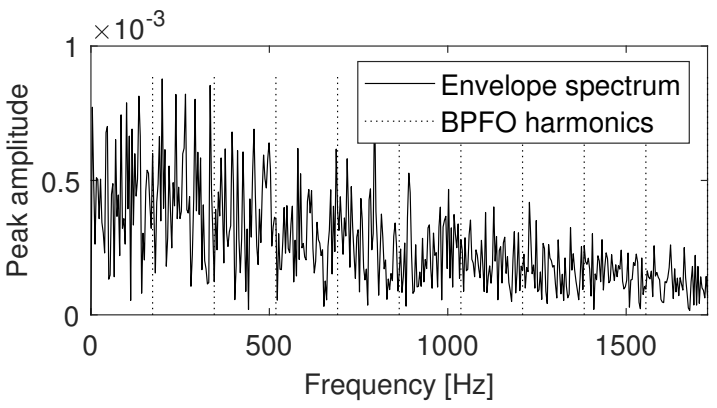

(B) . Pre-processing with OELMD method.

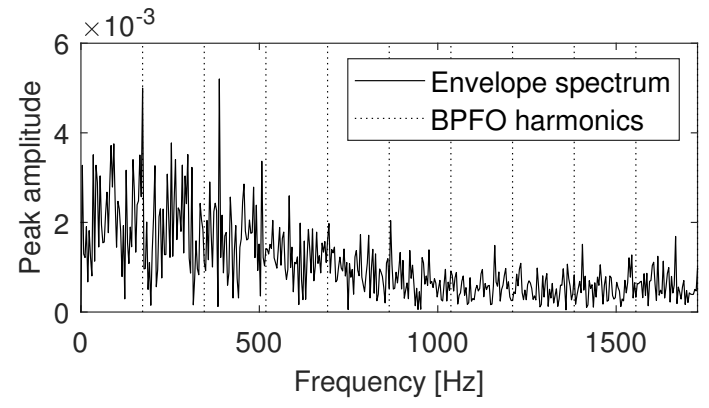

(C) . Pre-processing with proposed method.

FIGURE 13. Envelope spectrums of PF2 from bearing with outer race fault.

on being a simple and complementary solution to the algorithm proposed by [4].

Subsequently, a method for improving the decomposition results was proposed, based on the use of the relative-root-mean-square error and re-application of the decomposition to the obtained product functions. Regarding this section, the analyses of the results lead to the following conclusions:

(1.) The proposed method presented superior results in the mitigation of mode mixing as compared to the OELMD.

(2.) The execution of the method does not significantly increase the computational costs nor the processing time, since after the execution of the ensemble local mean decomposition - which is more time consuming - only the $L M D$, which presents a faster processing, is executed.

(3.) The excellent results obtained were achieved from synthetic signals and a small sample of real vibration signals, so the effectiveness of the method was not tested on highly complex signals. 


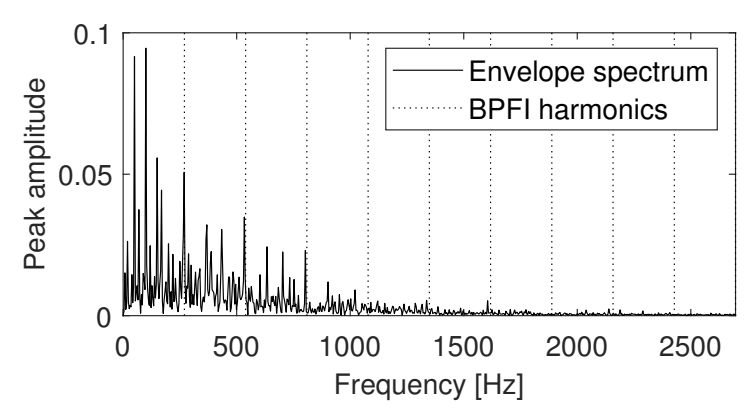

(A) . Without any pre-processing method.

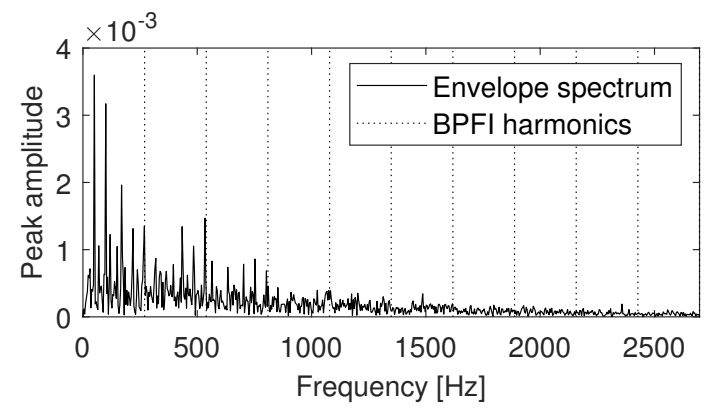

(B) . Pre-processing with OELMD method.

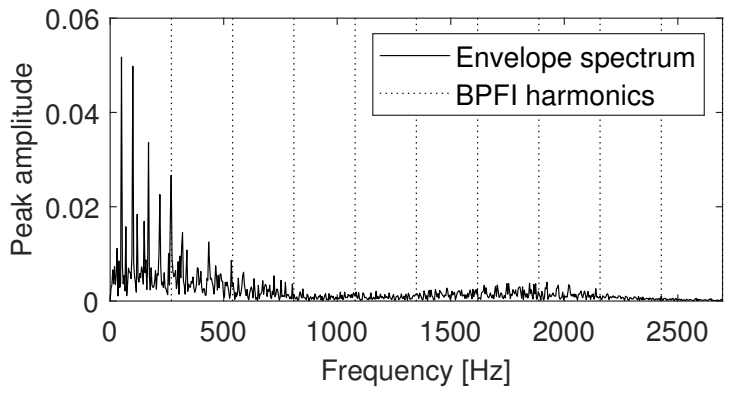

(c) . Pre-processing with proposed method.

Figure 14. Envelope spectrums of PF2 from bearing with inner race fault.

Finally, the effectiveness of the method was analysed for only one of its applications. That's why the proposed technique was tested against OELMD. In this section, the following conclusions can be drawn:

(1.) Although defects in bearing races require a simpler analysis, a slight improvement in the envelope spectrum was observed as compared to the ones derived from the ensemble local mean decomposition.

(2.) Spectral analysis does not always guarantee the detection of a bearing defect. This work has shown the effectiveness of a well-known meta-heuristic method in the optimization of an algorithm used for signal processing, proposing a new technique with a notable improvement in pre-processing the vibration signals and spectral analysis.

\section{ACKNOWLEDGEMENTS}

The authors greatly acknowledge the grant support from the Universidade Estadual do Oeste do Paraná.

\section{REFERENCES}

[1] N. E. Huang, Z. Shen, S. R. Long, et al. The empirical mode decomposition and the Hilbert spectrum for nonlinear and non-stationary time series analysis. Royal Society of London Proceedings Series A 454(1):903-995, 1996. https://doi.org/10.1098/rspa.1998.0193.

[2] J. S. Smith. The local mean decomposition and its application to EEG perception data. Journal of The Royal Society Interface 2(5):443-454, 2005. https://doi.org/10.1098/rsif.2005.0058.

[3] Y. Yang, J. Cheng, K. Zhang. An ensemble local means decomposition method and its application to local rub-impact fault diagnosis of the rotor systems. Measurement 45(3):561-570, 2012. https://doi.org/10.1016/j.measurement.2011.10.010.

[4] C. Zhang, Z. Li, C. Hu, et al. An optimized ensemble local mean decomposition method for fault detection of mechanical components. Measurement Science and Technology 28(3):035102, 2017. https://doi.org/10.1088/1361-6501/aa56d3

[5] Y. Wang, Z. He, Y. Zi. A Comparative Study on the Local Mean Decomposition and Empirical Mode Decomposition and Their Applications to Rotating Machinery Health Diagnosis. Journal of Vibration and Acoustics 132(2):021010, 2010. https://doi.org/10.1115/1.4000770

[6] Z. Wang, J. Wang, W. Cai, et al. Application of an Improved Ensemble Local Mean Decomposition Method for Gearbox Composite Fault Diagnosis. Complexity 2019, 2019. https://doi.org/10.1155/2019/1564243

[7] Y. Cheng, D. Zou. Complementary ensemble local means decomposition method and its application to rolling element bearings fault diagnosis. Proceedings of the Institution of Mechanical Engineers, Part O: Journal of Risk and Reliability 233(5):868-880, 2019. https://doi.org/10.1177/1748006X19838129.

[8] I. Bruant, L. Gallimard, S. Nikoukar. Optimal piezoelectric actuator and sensor location for active vibration control, using genetic algorithm. Journal of Sound and Vibration 329(10):1615-1635, 2010. https://doi.org/10.1016/j.jsv.2009.12.001

[9] L. B. Jack, A. K. Nandi. Genetic algorithms for feature selection in machine condition monitoring with vibration signals. IEE Proc-Vis Image Signal Process 147(3):205212, 2000. https://doi.org/10.1049/ip-vis:20000325

[10] H. Hao, Y. Xia. Vibration-based Damage Detection of Structures. Journal of Computing in Civil Engineering 16(3):222-229, 2002. https://doi.org/10.1061/(ASCE)08873801(2002)16:3(222)

[11] A. A. Adewuya. New Methods in Genetic Search with Real-Valued Chromosomes. Master thesis, Massachusetts Institute of Technology, 1996.

[12] Z. Michalewicz. Genetic Algorithms + Data Structures = Evolution Programs. Springer Berlin Heidelberg, Berlin, Heidelberg, 1996. https://doi.org/10.1007/978-3-662-03315-9

[13] R. L. Haupt, S. E. Haupt. Practical Genetic Algorithms. John Wiley \& Sons, Inc., Hoboken, NJ, USA, 2nd edn., 2003. https://doi.org/10.1002/0471671746 
[14] J. H. Holland. Adaptation in Natural and Artificial Systems: An Introductory Analysis with Applications to Biology, Control and Artificial Intelligence. Cambridge, MA, 1992. https://doi.org/10.1086/418447.

[15] J. H. Holland. Genetic Algorithms and the Optimal Allocation of Trials. SIAM Journal on Computing 2(2):88-105, 1973. https://doi.org/10.1137/0202009

[16] W. Guo, P. W. Tse. A novel signal compression method based on optimal ensemble empirical mode decomposition for bearing vibration signals. Journal of Sound and Vibration 332(2):423-441, 2013. https://doi.org/10.1016/j.jsv.2012.08.017

[17] Y. Wang, Z. He, Y. Zi. A demodulation method based on improved local mean decomposition and its application in rub-impact fault diagnosis. Measurement Science and Technology 20(2), 2009. https://doi.org/10.1088/0957-0233/20/2/025704

[18] F. D. D. M. Borges. Comparação de métodos de tratamento de sinais aplicáveis ao diagnóstico de defeitos em mancais de rolamento. Mestrado, Universidade Estadual do Oeste do Paraná, 2018.

[19] Z. Liu, M. J. Zuo, Y. Jin, et al. Improved local mean decomposition for modulation information mining and its application to machinery fault diagnosis. Journal of Sound and Vibration 397:266-281, 2017. https://doi.org/10.1016/j.jsv.2017.02.055

[20] G. Rilling, P. Flandrin, P. Gonçalvès. On empirical mode decomposition and its algorithms. In 6 th IEEE-EURASIP Workshop on Nonlinear Signal and Image Processing. Grado, Italy, 2003. https://doi.org/10.1210/en.2002-220356.
[21] L. Wang, Z. Liu, Q. Miao, X. Zhang. Time-frequency analysis based on ensemble local mean decomposition and fast kurtogram for rotating machinery fault diagnosis. Mechanical Systems and Signal Processing 103:60-75, 2018. https://doi.org/10.1016/j.ymssp.2017.09.042

[22] Y. Li, X. Liang, Y. Yang, et al. Early Fault Diagnosis of Rotating Machinery by Combining Differential Rational Spline-Based LMD and K-L Divergence. IEEE Transactions on Instrumentation and Measurement 66(11):3077-3090, 2017. https://doi.org/10.1109/TIM.2017.2664599.

[23] L. Deng, R. Zhao. An improved spline-local mean decomposition and its application to vibration analysis of rotating machinery with rub-impact fault. Journal of Vibroengineering 16(1):414-433, 2014.

[24] Y. Li, M. Xu, Z. Haiyang, et al. A new rotating machinery fault diagnosis method based on improved local mean decomposition. Digital Signal Processing 46:201-214, 2015. https://doi.org/10.1016/j.dsp.2015.07.001

[25] L. Wang, Z. Liu, Q. Miao, X. Zhang. Complete ensemble local mean decomposition with adaptive noise and its application to fault diagnosis for rolling bearings. Mechanical Systems and Signal Processing 106:24-39, 2018. https://doi.org/10.1016/j.ymssp.2017.12.031

[26] J. Sun, Z. Peng, J. Wen. Leakage aperture recognition based on ensemble local mean decomposition and sparse representation for classification of natural gas pipeline. Measurement 108:91-100, 2017. https://doi.org/10.1016/j.measurement.2017.05.029 